\title{
Inhibition of Nek2 by Small Molecules Affects Proteasome Activity
}

\author{
Lingyao Meng, ${ }^{1}$ Kent Carpenter, ${ }^{2}$ Alexis Mollard, ${ }^{1}$ Hariprasad Vankayalapati, ${ }^{1}$ \\ Steven L. Warner, ${ }^{2}$ Sunil Sharma, ${ }^{1,3}$ Guido Tricot, ${ }^{4}$ Fenghuang Zhan, ${ }^{4}$ and David J. Bearss ${ }^{2}$ \\ ${ }^{1}$ Center for Investigational Therapeutics, Huntsman Cancer Institute, Salt Lake City, UT 84112, USA \\ ${ }^{2}$ Tolero Pharmaceuticals Inc., 2975 Executive Parkway, Suite 320, Lehi, UT 84043, USA \\ ${ }^{3}$ Division of Medical Oncology, University of Utah, Salt Lake City, UT 84312, USA \\ ${ }^{4}$ Division of Hematology, Oncology, and Blood and Marrow Transplantation, Department of Internal Medicine, \\ University of Iowa, Iowa City, IA 52242, USA
}

Correspondence should be addressed to David J. Bearss; dbearss@toleropharma.com

Received 11 March 2014; Accepted 18 July 2014; Published 17 September 2014

Academic Editor: Maurizio Zangari

Copyright (C) 2014 Lingyao Meng et al. This is an open access article distributed under the Creative Commons Attribution License, which permits unrestricted use, distribution, and reproduction in any medium, provided the original work is properly cited.

Background. Nek2 is a serine/threonine kinase localized to the centrosome. It promotes cell cycle progression from G2 to M by inducing centrosome separation. Recent studies have shown that high Nek2 expression is correlated with drug resistance in multiple myeloma patients. Materials and Methods. To investigate the role of Nek2 in bortezomib resistance, we ectopically overexpressed Nek2 in several cancer cell lines, including multiple myeloma lines. Small-molecule inhibitors of Nek2 were discovered using an inhouse library of compounds. We tested the inhibitors on proteasome and cell cycle activity in several cell lines. Results. Proteasome activity was elevated in Nek2-overexpressing cell lines. The Nek2 inhibitors inhibited proteasome activity in these cancer cell lines. Treatment with these inhibitors resulted in inhibition of proteasome-mediated degradation of several cell cycle regulators in HeLa cells, leaving them arrested in G2/M. Combining these Nek2 inhibitors with bortezomib increased the efficacy of bortezomib in decreasing proteasome activity in vitro. Treatment with these novel Nek2 inhibitors successfully mitigated drug resistance in bortezomib-resistant multiple myeloma. Conclusion. Nek2 plays a central role in proteasome-mediated cell cycle regulation and in conferring resistance to bortezomib in cancer cells. Taken together, our results introduce Nek 2 as a therapeutic target in bortezomibresistant multiple myeloma.

\section{Introduction}

Nek2 is a serine/threonine protein kinase, belonging to the Nek family of cell cycle regulators [1]. The first member of this family, NIMA, was originally identified as a mutant preventing $A$. nidulans cells from entering mitosis. Thus, "NIM" stands for "never in mitosis" [2]. The Nek family has 11 members (Nek1-11), and Nek2 is the one with the highest sequence identity compared to NIMA [1]. Modern biochemical and proteomic data has shown that Nek2 is a core component of the human centrosome, and similar findings have also been reported for homologues of Nek2 in Drosophila, Xenopus, and mouse [3-6]. There is substantial evidence that Nek2 plays a key role in centrosome separation and promotion of the cell cycle from G2 to M phase [710]. Because the ubiquitin-proteasome system has been previously targeted with the proteasome inhibitor bortezomib in breast cancer [11-13], a few groups began to study and have subsequently reported that Nek2 regulates cell cycle progression in breast cancer cell lines [14-16].

While the effectiveness of proteasome inhibition in breast cancer continues to be evaluated, bortezomib continues to be a mainstay treatment for relapsed refractory MM $[17,18]$. In spite of bortezomib's usefulness in improving overall survival in some patients, as many as one-third of relapsed MM patients do not respond to bortezomib and those that do respond often develop resistance [18-20]. For this reason, we sought to identify those genes related to myeloma drug 
resistance and disease relapse in a previous report. Among the genes studied, we found that Nek 2 most accurately predicted poor prognosis, cell proliferation, and drug resistance in ex vivo and in vitro models of multiple myeloma [21].

Although several groups have tried to validate Nek2 as a therapeutic target using both small molecules and siRNA, few of them actually achieved efficient inhibition of Nek2 by small molecules [16, 22-25]. In this study, we identify a series of potent and selective inhibitors of Nek2, derived from a kinase-focused library screening approach. This approach provided us with selective, orally available small molecule inhibitors of Nek2, including HCI-2184, HCI2388, and HCI-2389. All three of the compounds are related and have a pyrimidine scaffold as their core pharmacophore. These compounds inhibited proteasome activity in vitro and mitigated bortezomib resistance induced by Nek2 overexpression. Taken together, the data suggest that Nek2 plays an important role in the uncontrolled proliferation of MM cells and introduces Nek2 as a therapeutic target in relapsed refractory MM cells resistant to bortezomib.

\section{Materials and Methods}

2.1. Generation of Stable Nek2 Overexpressing (OE) Cell Lines. The Nek2 coding sequence was purchased and subcloned from a pCMV6-Entry vector (OriGene). Restriction enzymes AsiSI and XhoI were used to ligate the NEK2 gene into the pCMV6-GFP vector (OriGene). The correct sequence of pCMV6-NEK2-GFP was verified by sequencing. Plasmid was generated in Top 10 cells (Invitrogen) and the plasmid was purified using the Small Scale Plasmid DNA Purification Kit (QIAGEN). Purified pCMV6-NEK2-GFP was used to transfect HeLa cells in 6-well plates, using Lipofectamine 2000 (Invitrogen). We chose to transfect HeLa cells with the pCMV6-NEK2-GFP plasmid because a previous report indicated the successful transfection of plasmids into NT2/D1 and HeLa cells using Lipofectamine 2000 without visible toxicity [26]. The final concentration of plasmid was $0.4 \mu \mathrm{g} / \mathrm{mL}$ and the cell density was $8 \times 10^{5}$ cells per well. G418 (Invitrogen) was added to DMEM medium with final concentration of $1 \mathrm{mg} / \mathrm{mL}$. HeLa cells were cultured in G418 containing medium for one month. Clones were then isolated and continuously cultured as stable Nek2 OE HeLa cells. The same process was conducted using the pCMV6-GFP vector to establish stable GFP OE HeLa cells.

Other Nek2 OE multiple myeloma cell lines, including ARP1, H929, and KMS28PE cells, were developed as described in our previous report [21]. As with the HeLa cells, the final concentration of plasmid was $0.4 \mu \mathrm{g} / \mathrm{mL}$ and the cell density was $8 \times 10^{5}$ cells per well. Three additional clones of the ARP-1 cell line, Nek2-OE, Nek2-knockdown (KD), and bortezomib-resistant lines were generated as described in our previous report [21].

2.2. Western Blot Analysis. Following the indicated treatments, cells were washed by cold $1 \mathrm{X}$ PBS buffer and were lysed using NP-40 cell lysis buffer (Life Technology). Wholecell lysates were prepared and subjected to Western blot analysis as described in our previous report [21]. Briefly, after incubation with primary antibodies (Cell Signaling), the blots were probed with HRP-secondary antibodies (abcam). The blots were then detected with an ECL Detection Kit (Amersham).

\subsection{Proteasome Isolation and In Vitro Proteasome Activ-} ity Assays. The $26 \mathrm{~S}$ proteasome was isolated from wholecell lysates by ultracentrifugation as previously described [27]. Proteasome activity was tested either in 96-well plates or 384-well plates using the Proteasome-Glo Trypsin-Like Assay (Promega). The assay was performed according to the vendor's protocol, and the proteasome concentration was optimized to $0.25 \mu \mathrm{g} / \mathrm{mL}$.

2.4. In Vitro Nek2 Inhibition Assays. Compounds were incubated with human Nek2 kinase (Invitrogen) and then kinase activity was examined by the Kinase-Glo Luminescence Kinase Assay (Promega). The assay was performed according to the manufacturer's protocol in 384-well plates' format using $60 \mathrm{mM}$ Nek2. Twelve different concentrations were set for each compound: $100 \mu \mathrm{M}, 30 \mu \mathrm{M}, 10 \mu \mathrm{M}, 3 \mu \mathrm{M}, 1 \mu \mathrm{M}$, $300 \mathrm{nM}, 100 \mathrm{nM}, 30 \mathrm{nM}, 10 \mathrm{nM}, 3 \mathrm{nM}, 1 \mathrm{nM}$, and $0.3 \mathrm{nM}$.

2.5. Cell Viability Assays. Cell viability was determined using the ATPlite 1Step Kit (PerkinElmer) in 96-well plates. The assay was performed according to the vendor's protocol. Cell viability was assessed by measuring live cell ATP activity.

2.6. Cell Cycle Analysis. Cell cycle analysis was performed as described [28]. HeLa cells were harvested and resuspended in Krishan's Buffer $(0.1 \%$ sodium citrate, $50 \mu \mathrm{g} / \mathrm{mL}$ propidium iodide, $20 \mu \mathrm{g} / \mathrm{mL}$ RNase A, and 0.5\% NP-40). Flow cytometry was conducted on a FACScan cytometer (Becton, Dickinson and Company). Collected data was analyzed by FlowJo 6.0b software (Tree Star, Inc.).

2.7. Statistical Analyses. Data was tested for statistical significance by unpaired $t$-tests using the Graph-Pad InStat Software. Data was considered statically significant when $P<$ 0.05 .

\section{Results}

3.1. Nek2 Overexpression Induced Bortezomib Resistance in HeLa Cells. We previously reported that bortezomib resistance is accompanied with Nek2 upregulation in MM patients [21]. To confirm this correlation, we used the constructed Nek2-GFP plasmid to transfect HeLa cells, and Nek2 overexpression was first confirmed by Western blot (Figure 1(a)). The lower band in the blots corresponds to endogenous Nek2 whereas the larger band corresponds to the Nek2GFP plasmid. Increased phosphorylation of PP1- $\alpha$, a known substrate of Nek2 [29], was also verified by Western blot in Nek2-OE cells (Figure 1(b)).

The two most viable HeLa Nek2-OE clones and HeLa GFP-OE clones were selected for the following experiments. Bortezomib was used to treat these HeLa cells in a 96-well 


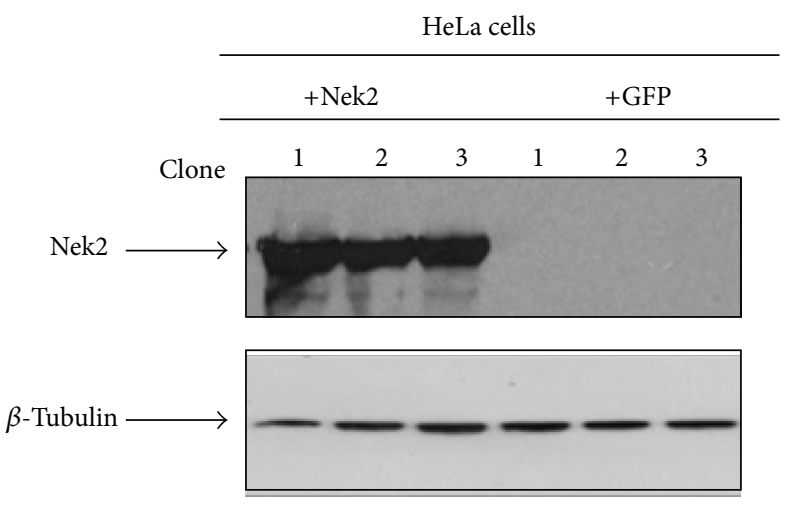

(a)

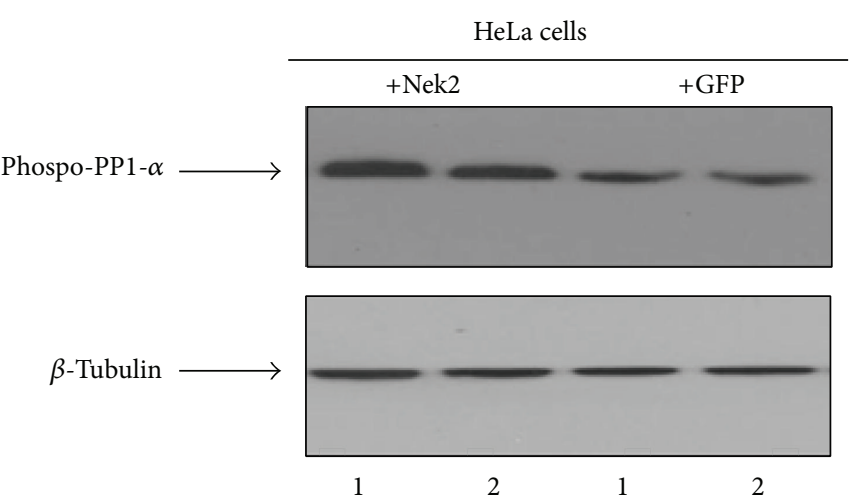

(b)

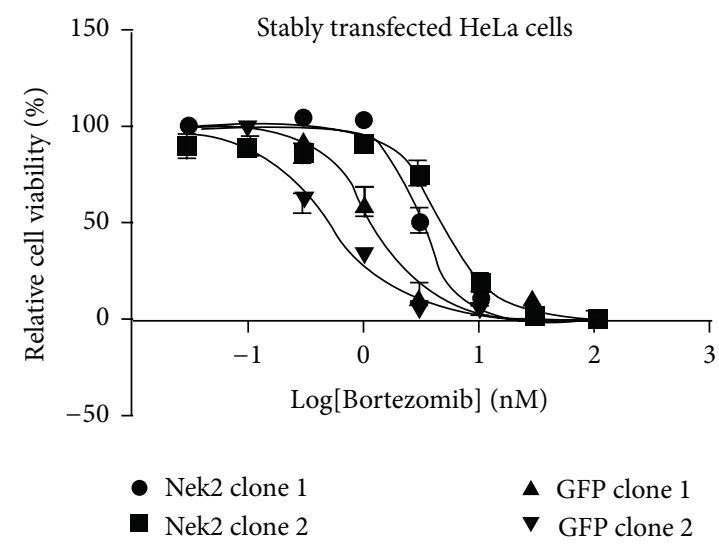

(c)

FIGURE 1: Nek2 overexpression causes HeLa cells to become resistant to bortezomib. (a) The Nek2 gene was cloned into a GFP expression vector as described in Materials and Methods Section. HeLa cells were then transfected with either the Nek2-GFP plasmid or GFP expression vector alone. Anti-NEK2 antibody was used to confirm NEK2 overexpression as determined by Western blot. (b) Nek2 overexpression increased the level of phosphorylated PP1- $\alpha$ in the two surviving Nek2 transfected clones. (c) Nek2-GFP transfected HeLa cells were resistant to bortezomib treatment compared to GFP-transfected clones. Bortezomib was used to treat HeLa cells with the concentration range from $100 \mathrm{nM}$ to $0.03 \mathrm{nM}$. Within this range, at any given concentration of bortezomib, Nek2-transfected clones yielded higher cell viability than GFP-transfected clones.

plate under different concentrations $(100 \mathrm{nM}, 30 \mathrm{nM}, 10 \mathrm{nM}$, $3 \mathrm{nM}, 1 \mathrm{nM}, 0.3 \mathrm{nM}, 0.1 \mathrm{nM}$, and $0.03 \mathrm{nM}$ ) with $0.1 \%$ DMSO as control. After 72 hours, cell viability was examined by the ATP lite assay. At every concentration of bortezomib, Nek2-OE clones yielded higher cell viability than GFP clones (Figure 1(c)). These data suggest that bortezomib resistance was induced by Nek2 overexpression in HeLa cells, which is consistent with our previously reported data [21].

3.2. Proteasome Activity Was Significantly Increased by Nek2 Overexpression. Because bortezomib is able to target cancer cells by proteasome inhibition [30], we hypothesized that Nek2 overexpression would increase proteasome activity in transfected cells and subsequently confer bortezomib resistance. To test this hypothesis, the $26 \mathrm{~S}$ proteasome was isolated by ultracentrifugation from the stable Nek2-OE cells. Three different human MM cell lines, including ARP1, H929, and KMS28PE, were tested. Among them, we tested four verified clones of the ARP-1 cell line, including wild-type, Nek2-OE, Nek2-knockdown (KD), and bortezomib-resistant clones.
These cell lines were generated and verified as described in our previous report [21].

In vitro proteasome activity from the isolated proteasome was tested by the Proteasome-Glo Trypsin-Like Assay. For all the studied cell lines, the proteasome activity of the Nek2OE cells was significantly higher than the control (GFPtreated for HeLa cells and empty vector treated cells for H929, KMS28PE, and ARP-1 cells). Bortezomib resistant ARP-1 cells exhibited the highest proteasome activity (Figures 2(a)-2(d)). These results support our hypothesis and imply that Nek2 overexpression is one of the mechanisms behind increased proteasome activity in bortezomib-resistant MM cell lines.

3.3. Nek2 Inhibitors Reduce the In Vitro Proteasome Activity in Nek2-Expressing Cell Lines. A focused screening library of 2000 compounds was assembled from an in-house collection of previously synthesized kinase inhibitors utilizing a single concentration screening approach in a Nek2 biochemical kinase assay. This yielded four compounds with Nek2 kinase inhibition greater than $80 \%$ at $10 \mu \mathrm{M}$. These 4 hits were 


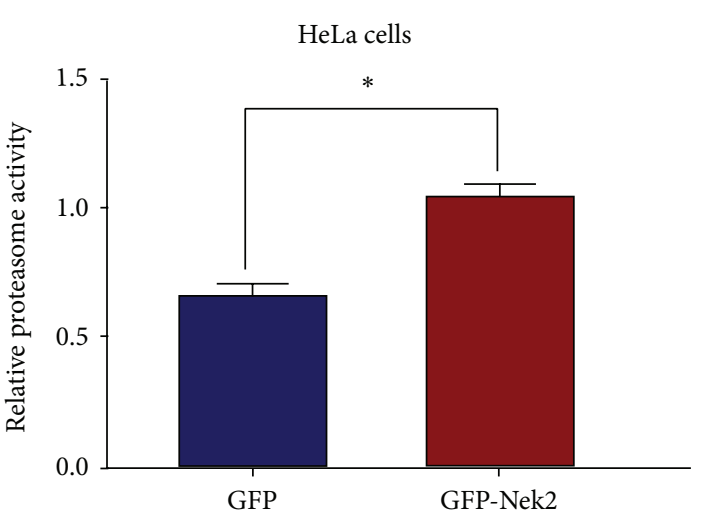

(a)

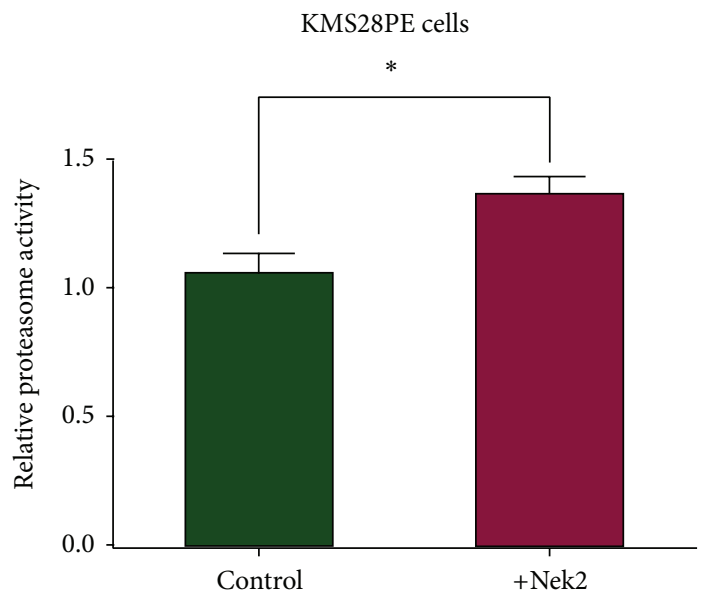

(c)

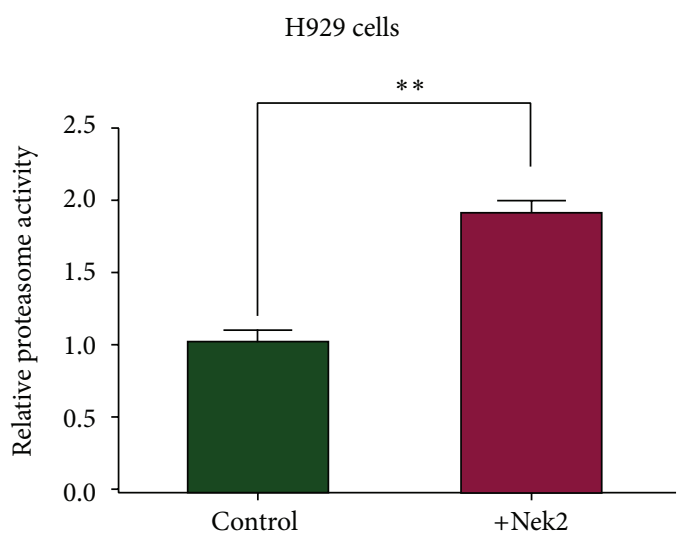

(b)

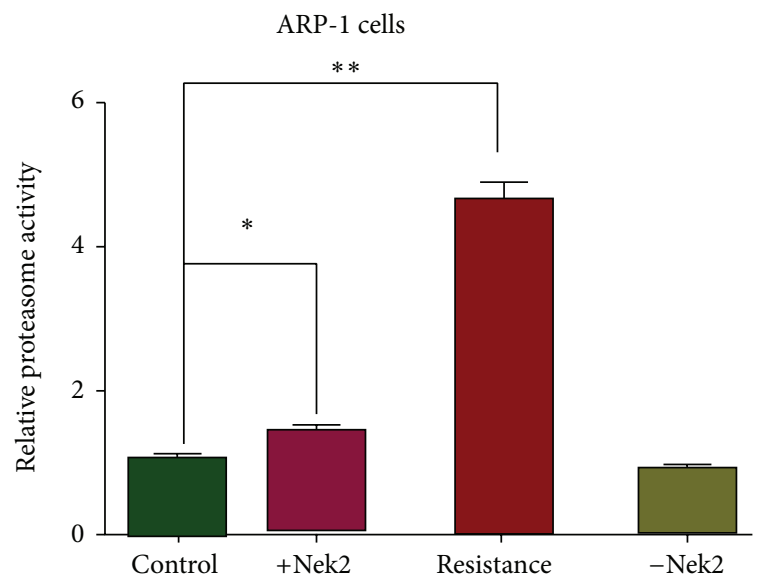

(d)

FIGURE 2: Nek2 overexpression elevates the proteasome activity in multiple cancer cell lines. (a) Proteasome activity is significantly increased in Nek2 overexpressed HeLa cells compared to GFP-transfected control. Proteasome activity was also significantly elevated in H929 (b), KMS28PE (c), and ARP-1 (d) cell lines compared to empty vector transfected (control). For the ARP-1 cell line, Nek-2-OE, NEK-2-KD, and bortezomib-resistant clones were tested in addition to wild-type cells. The $26 \mathrm{~S}$ proteasome was isolated by ultracentrifugation and the proteasome activity was determined by Proteasome-Glo Assay. (d) For ARP1 cells, the bortezomib-resistant cells (third column in (d)) showed higher proteasome activity. For Figures $2(\mathrm{a})-2(\mathrm{~d}),{ }^{*} P<0.05,{ }^{* *} P<0.01$.

filtered by physical property calculations, in vitro ADME, and kinase selectivity filters to give one compound, HCI2184, that was selected for further experiments examining the role of Nek2 in drug resistance. Using the KinaseGlo assay, we determined the $\mathrm{IC}_{50}$ of HCI-2184 and found that it was $<100 \mathrm{nM}$. Structure-based optimization was used to synthesize additional analogues of HCI-2184 and three compounds were selected as potential leads, HCI-2184, HCI2388 , and HCI-2389, all of which yield an average $\mathrm{IC}_{50}$ under $50 \mathrm{nM}$ (Figures 3(a)-3(c)). Among them, HCI-2389 was the most potent Nek2 inhibitor. This is most likely due to its irreversible binding mode of action (see Supplementary Figure S1 available online at http://dx.doi.org/10.1155/2014/273180) which was tested by the Kinase-Glo assay with the drug preincubated with Nek2. The Nek2 inhibitory activity of HCI2389 was significantly increased after preincubation times as short as 0.5 hours (Figure 3(d)).

Based on its potency, HCI-2389 was selected to treat the Nek2-OE HeLa cells. We performed Western blots to measure the downstream effects of Nek2 inhibition caused by HCI2389 treatment. We found that the level of phosphorylated PP1- $\alpha$ was significantly decreased in HeLa cells treated with concentrations as low as $10 \mathrm{nM}$ of HCI-2389 for 72 hours (Figure 3(e)).

Our observation that Nek2 overexpression increased proteasome activity led us to ask whether our Nek2 inhibitors were able to inhibit this increased activity. We tested this hypothesis by isolating the $26 \mathrm{~S}$ proteasome by ultracentrifugation from multiple Nek2-OE cells as described in Materials and Methods. Interestingly, Nek2 was found to be involved in the proteasome complex (Figure 4(a)), suggesting a possible direct interaction with the proteasome components. In accordance with this notion, the levels of Nek2 associated with the proteasome were proportional to overall Nek2 levels in cells (Figure 4(a)). It is important to note this relationship was not as clear in the KMS28PE cell line, where levels of both endogenous and transfected Nek2 were not as apparent (Figure 4(a)). To test the effect of our Nek2 inhibitors on 
<smiles>CN1CCN(c2ccc(Nc3ncc(Cl)c(Nc4ccccc4S(=O)(=O)N(C)C)n3)cc2)CC1</smiles>

HCI-2184

$\mathrm{IC}_{50}=39.90 \mathrm{nM}$ (8 runs)

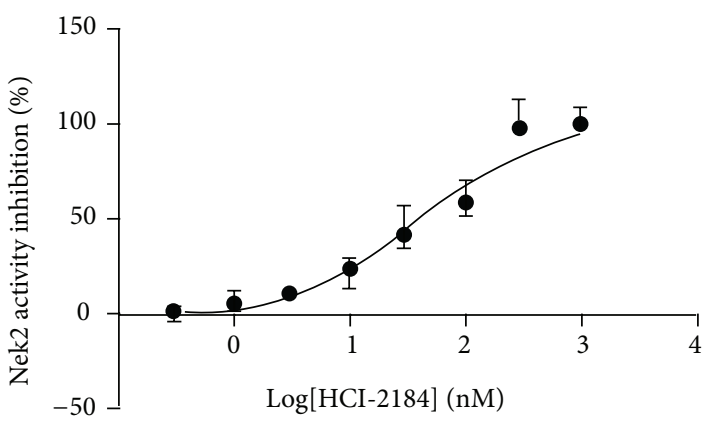

(a)<smiles>C=CC(=O)Nc1cc(Nc2ncc(Cl)c(Nc3ccccc3S(=O)(=O)N(C)C)n2)ccc1N1CCN(C)CC1</smiles>

HCI-2389

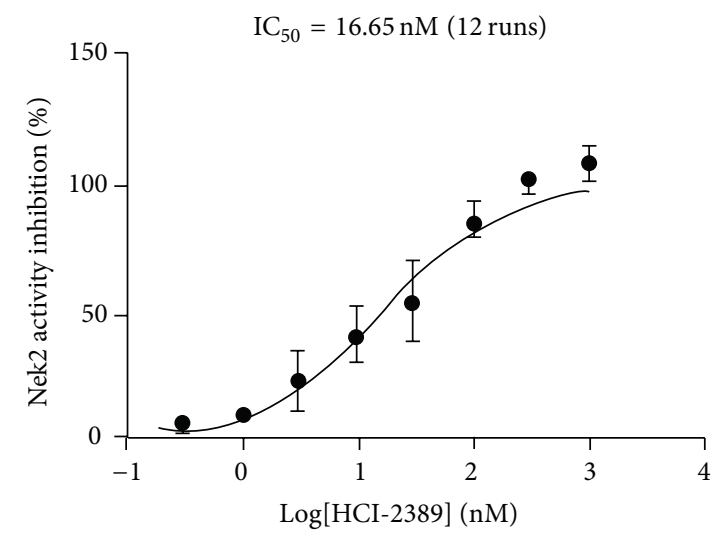

(c)<smiles>CN1CCN(c2ccc(Nc3ncc(Cl)c(Nc4ccccc4S(=O)(=O)N(C)C)n3)cc2N)CC1</smiles>

HCI-2388

$\mathrm{IC}_{50}=34.27 \mathrm{nM}$ (8 runs)

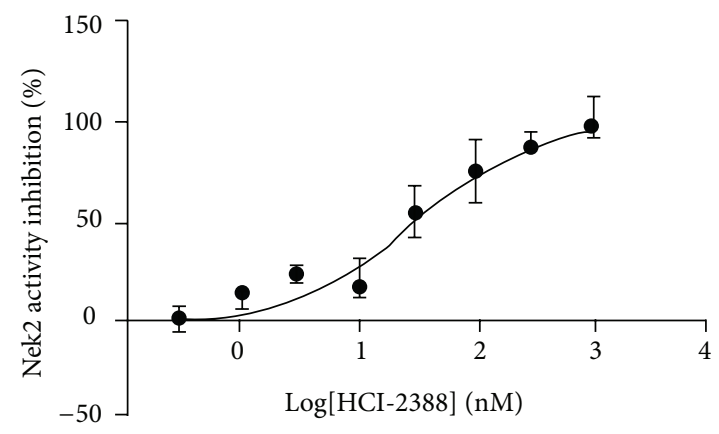

(b)

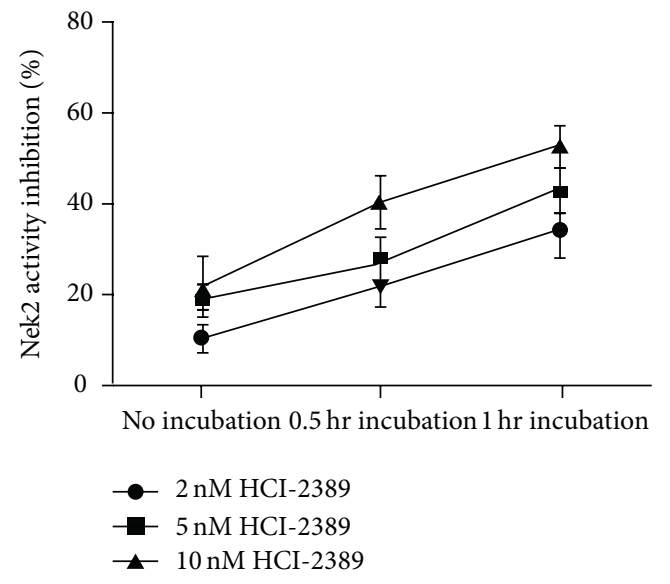

(d)

Figure 3: Continued. 

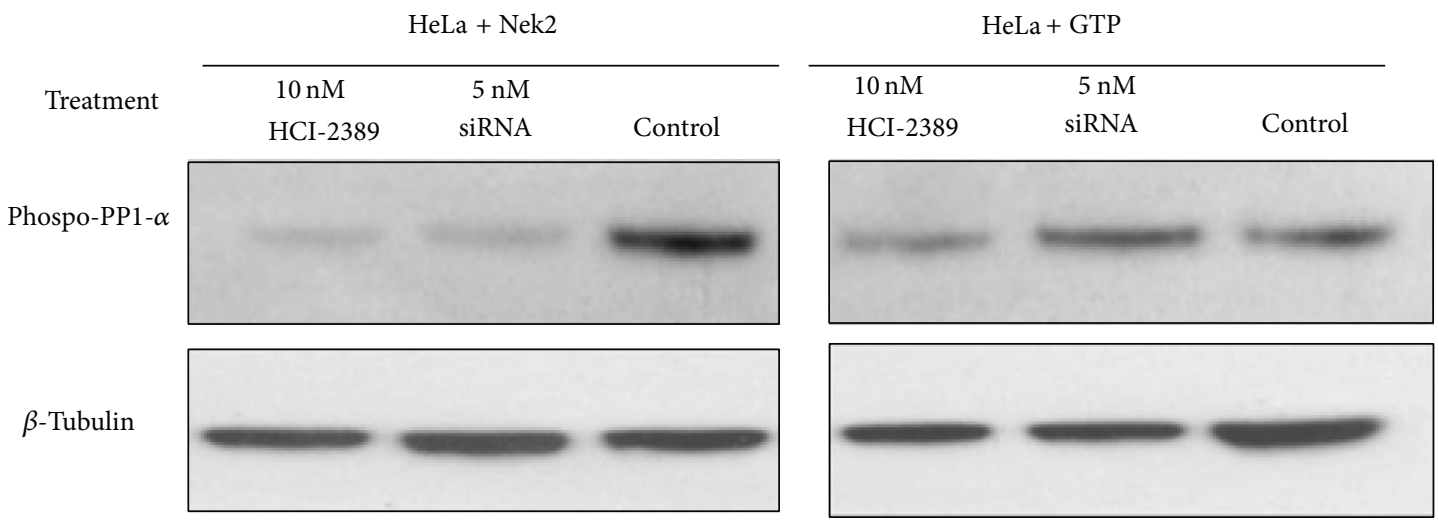

(e)

FIGURE 3: Novel Nek2 Inhibitors significantly Inhibit Nek2's activity. (a), (b), and (c), three compounds, HCI-2184, HCI-2388, and HCI-2389 were designed by virtual screening. Synthesized compounds were validated by NMR and MS. The abilities of the three compounds to inhibit Nek2 kinase were tested by Kinase-Glo Assay. (d) HCI-2389 acts as an irreversible Nek2 inhibitor. A 0.5 hr incubation of HCI-2389 and Nek2 kinase increased the ability of HCI-2389 to inhibit Nek2. This effect was more pronounced when HCI-2389 was incubated with Nek2 kinase for $1 \mathrm{hr}$. (e) $10 \mathrm{nM}$ HCI-2389 treatment for 72 hours greatly decreased the level of phosphorylated PP1- $\alpha$ in both Nek2 overexpressed HeLa cells and GFP controls. The effect was equal to or greater than treatment with $5 \mathrm{nM} \mathrm{Nek-2}$ siRNA.

proteasome activity, the compounds were incubated with the isolated proteasome followed by the Proteasome-Glo assay. Our Nek2 inhibitors inhibited proteasome activity in vitro at a level similar to bortezomib (Figures 4(b)-4(e)). Based on these results, we concluded that the Nek2 inhibitors were responsible for the decrease in proteasome activity in the Nek2-OE cancer cell lines tested.

We further studied the effect of Nek2 inhibitors on additional cell lines using the ATP-lite cell viability assay. We treated a large panel of cell lines $(n=36)$ with our Nek2 inhibitors. These 36 cell lines were either responsive ("responsive" was defined as an $\mathrm{IC}_{50}$ value of less than $1 \mu \mathrm{M}$ ) to both HCI-2184 and HCI-2389 or nonresponsive to either of the two inhibitors selected for the proteasome activity assay. Data analysis showed that although there was not a strict proportional relation between proteasome activity and Nek2 inhibitor responsiveness, the average proteasome activity of the sensitive cell lines was significantly higher than that of the nonsensitive cell lines (Figure 5).

\subsection{The Combination of Bortezomib and Nek2 Inhibitors} Reduces Proteasome Activity to a Greater Extent Than Either Drug Alone. We next combined our Nek2 inhibitors with bortezomib in the proteasome-Glo assay to determine whether Nek2 inhibitors could be used in combination with bortezomib. In treated HeLa cells, both HCI-2184 and HCI2389 significantly increased the effectiveness of bortezomib in inhibiting proteasome activity at concentrations as low as $10 \mathrm{nM}$ (Figure 6(a)). Additionally, dose response studies confirmed that these two Nek2 inhibitors shift the inhibition curve of bortezomib (Figure 6(b)).

For the other three cell lines studied, including H929, KMS28PE, and ARP1, HCI-2389 was also able to increase the efficacy of bortezomib, while HCI-2184 had less of a synergistic effect (Figures 6(c)-6(e)). Again, the irreversible binding of HCI-2389 provides a possible explanation for this difference between compounds.

These results provide evidence that proteasome activity can be inhibited to a greater extent when combining Nek2 inhibitors with bortezomib, compared to bortezomib alone, suggesting that Nek2 is a potential molecular target that might be used in combination with bortezomib to treat MM patients.

3.5. Nek2 Inhibitors Prevented Mitotic Proteins from Being Degraded by Proteasome, Causing $G_{2} / M$ Phase Arrest. Many proteins are targeted and degraded by the proteasome for mitotic entry as well as mitotic exiting [31-34]. Degradation of Cyclin B and Cdc2 plays a significant role in mitotic regulation [35-38]. Previous research has shown that downregulation of proteasome activity lead to the accumulation of Cyclin B [39], triggered by the overexpression of Hecl, a substrate of Nek2. As Nek2 overexpression elevated proteasome activity and Nek2 inhibition decreased it, we set out to evaluate the levels of a few key mitotic regulators targeted by the proteasome.

In this experiment, cells were synchronized in mitotic phase, followed by treatment of Nek2 inhibitors for 72 hours. The levels of Cyclin B and Cdc2 were then evaluated by Western blot. Both Cyclin B and Cdc2 were found to be downregulated by Nek2 overexpression (Figure 7(a)), which is consistent with the finding that Nek2 overexpression causes increased proteasome activity. Further, treating the cells with HCI-2389 or Nek2-siRNA successfully inhibited the degradation of Cyclin B and Cdc2. This effect was not as dramatic in the GFP controls, which expressed only basal levels of Nek2.

Cell cycle analysis was performed to examine the effect of Nek2 inhibition on the cell cycle. The stably transfected HeLa cells were treated with $10 \mathrm{nM}$ of HCI-2184 and HCI2389 for 24 hours and then analyzed by flow cytometry. We 

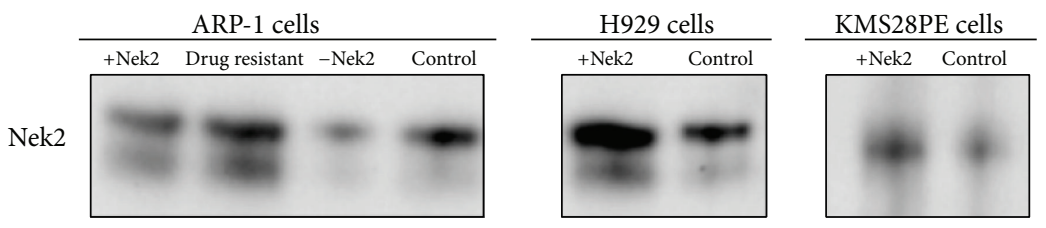

(a)

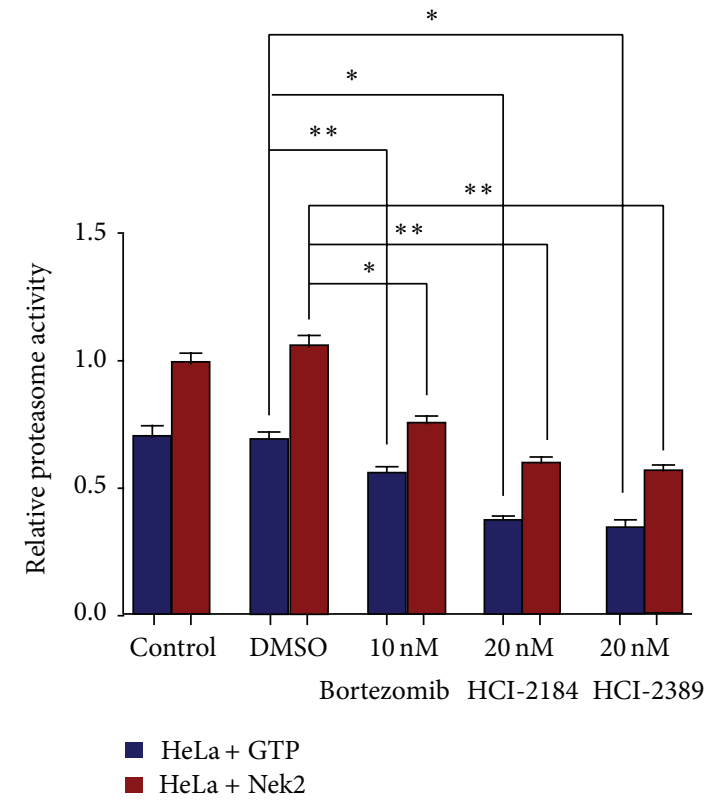

(b)

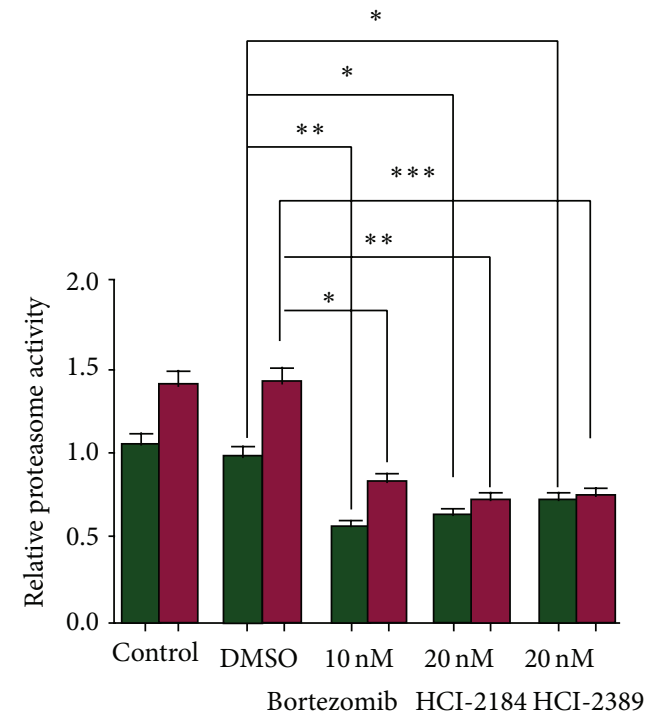

- KMS28PE control

- KMS28PE + Nek2

(d)

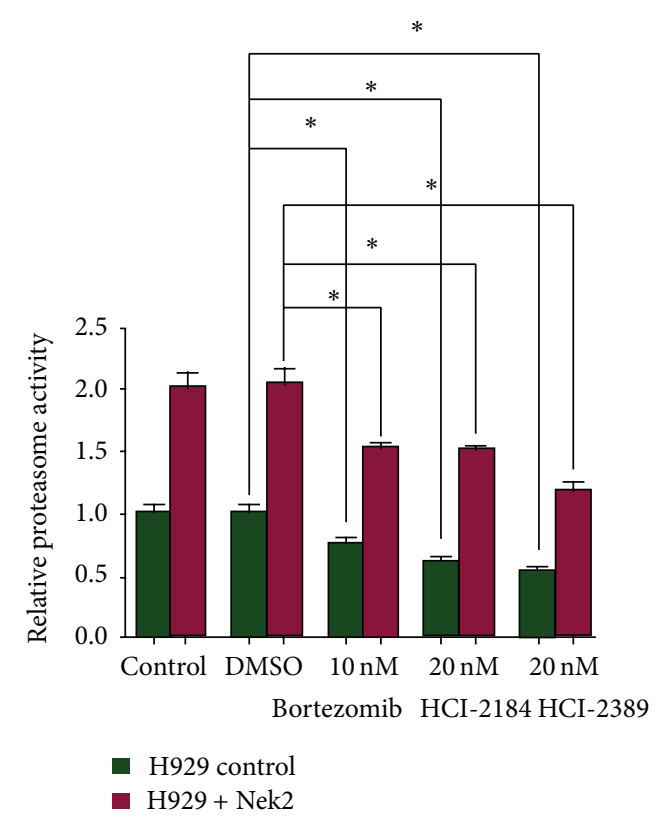

(c)

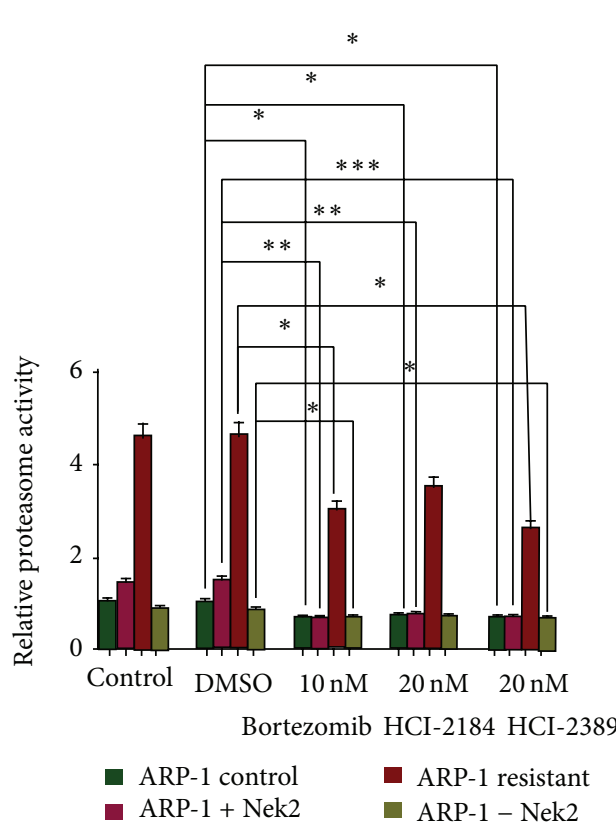

(e)

Figure 4: Novel Nek2 inhibitors effectively decrease the proteasome activity in vitro for multiple cancer cell lines. (a) Nek2 was found to be involved in the $26 \mathrm{~S}$ proteasome in cancer cell lines. The $26 \mathrm{~S}$ proteasome was isolated by ultracentrifugation and the presence of Nek2 in the $26 \mathrm{~S}$ proteasome was determined by Western blot. (b), (c), (d), and (e), Incubation of HCI-2184 and HCI-2389 significantly inhibits the proteasome activity for HeLa cells (b), H929 cells (c), KMS28PE cells (d), and ARP1 cells (e). (e) The irreversible Nek2 inhibitor HCI-2389 worked better than HCI-2184 in decreasing the proteasome activity for ARP1 cells that are resistant to bortezomib treatment. For Figures $4(\mathrm{~b})-4(\mathrm{e}),{ }^{*} \mathrm{P}<0.05,{ }^{* *} \mathrm{P}<0.001,{ }^{* * *} \mathrm{P}<0.0001$. 


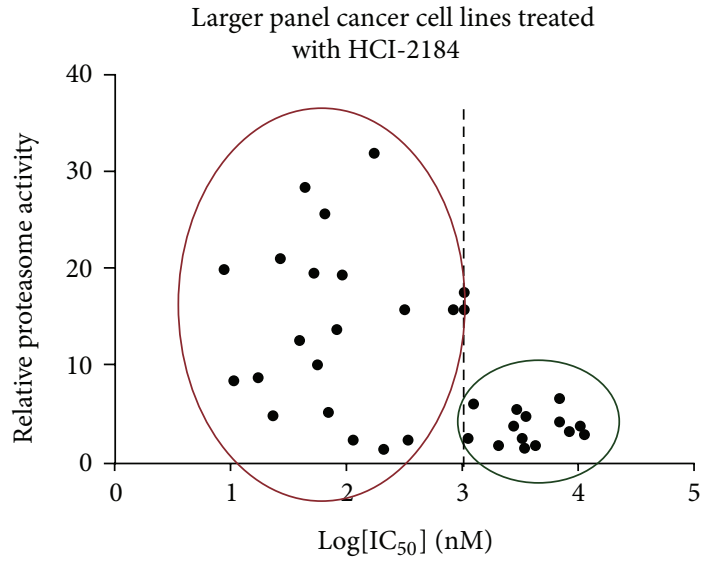

(a)

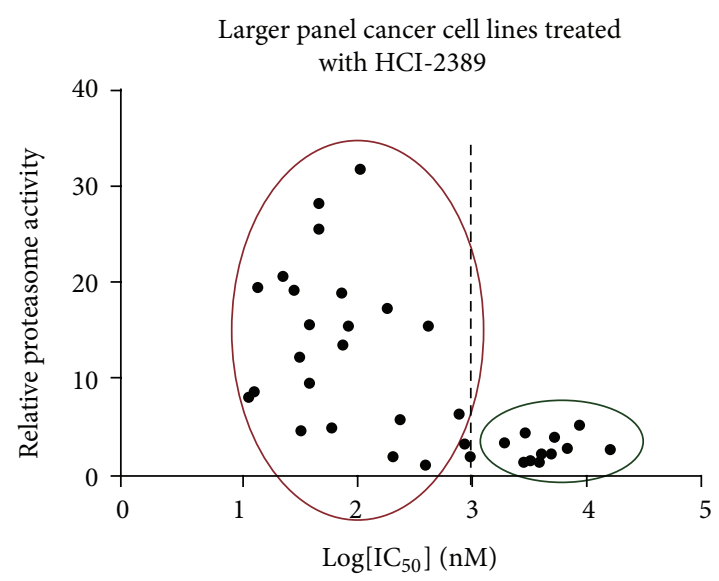

(b)

FIGURE 5: The sensitivity of cancer cell lines to Nek2 inhibitors is correlated with their proteasome activity. (a) Cell lines sensitive to HCI-2184 treatment had, on average, higher proteasome activity compared to resistant cell lines. (b) Cell lines sensitive to HCI-2389 treatment had, on average, higher proteasome activity compared to resistant cell lines. These cancer cell lines were selected from the 150 cell lines in our lab, based on whether or not they were sensitive to both HCI-2184 or HCI-2389. "Sensitive" was defined as an $\mathrm{IC}_{50}$ value of $1 \mu \mathrm{M}$ or lower. The $26 \mathrm{~S}$ proteasomes were isolated by ultracentrifugation and proteasome activity measured by Proteasome-Glo Assay.

found that almost $50 \%$ of the Nek2 inhibitor-treated cells were arrested in G2/M phase (Figure 7(b)). As before, HCI2184 did not work as well as HCI-2389 in arresting HeLa GFP OE cells, and this is probably because HCI-2389 is a more specific Nek2 inhibitor than HCI-2184. Further research will be needed to elucidate the detailed inhibitory mechanisms of these compounds.

In summary, Cyclin B and Cdc2 were downregulated by Nek 2 overexpression and Nek 2 inhibition reversed this effect. Nek2 inhibitors, through inhibition of proteasome activity, inhibited Cyclin B and Cdc2 from being degraded. This resulted in cell cycle arrest in $\mathrm{G}_{2} / \mathrm{M}$ phase in the Nek2-OE cells.

\section{Discussion}

Although progress in the treatment of MM has been made in the past decade $[40,41]$, myeloma remains largely incurable with current therapeutic strategies. Bortezomib is one of the most effective chemotherapies for MM, but drug resistance remains a crucial problem with bortezomib treatment [17, 19, 20]. Little is known about the molecular mechanisms involved in this resistance. In our previous report, we used gene expression profiling in a variety of MM cases and identified Nek2 as the most significant gene associated with early relapse [21]. Other reports have similarly shown that Nek2 overexpression induces chemotherapeutic resistance in vitro $[15,42]$. It is clear that there is an urgent need for exploring the mechanism linking the Nek2 kinase to drug resistance and the development of novel Nek2 inhibitors. To our knowledge, this study represents the first link connecting Nek2's biological function of regulating proteasome activity as the mechanism of bortezomib resistance in multiple myeloma. It is also the first to establish highly effective Nek2 inhibitors that successfully inhibit proteasome activity in cancer cell lines.
The 26S proteasome complex is a core component of the ubiquitin-proteasome system (UPS) of protein degradation. Ubiquitination regulates multiple cell cycle aspects including checkpoints control and cell growth progression [18, 43, 44]. The $26 \mathrm{~S}$ proteasome is essential for the rapid elimination of the cell cycle regulators and the transcription factors such as $\mathrm{NF}-\kappa \mathrm{B}$, whose fast degradation is important to the proper cell processes $[44,45]$. Cdk1 and Cdk2 drive progression through each cell cycle phase and G2/M transition in particular $[38,46]$. The activation of Cdks greatly depends on the availability of their cyclin partners, and cyclin levels are strongly regulated by the UPS [32]. In addition, the UPS has been shown to regulate the Cdk inhibitors such as Weel $[37,46]$. Studies have shown that two complexes are involved in the UPS regulation of cell cycle: the anaphase-promoting complex or cyclosome (APC/C) and the Skp1/Cullin-1/F-box protein complex (SCF) [34, 47, 48]. These two complexes have different cellular functions and play crucial roles in different cell phases. APC/C regulates the degradation of mitotic cyclins, such as Cyclin B1, and consequently inhibits Cdk1, leading cells to mitotic exit $[41,42]$.

Previous reports suggested that Nek2 primarily played a role in regulating centrosome separation [7-10]. Overexpression of active Nek2 induces premature splitting of centrosomes, while silencing of Nek2 blocks spindle and chromosome segregation. As centrosome separation is crucial for mitotic entry, Nek2 was thought to participate in cell cycle control. However, compared to other mitotic kinases, Nek2's function is relatively subtle and, in our study, neither suppression nor silencing of Nek2 expression dramatically affected the cell cycle. This has been the major obstacle for studying Nek2's biological function. In this research, we explored Nek2's function and we confirmed the correlation of Nek2 overexpression and bortezomib resistance in HeLa cells. Bortezomib exerts its effects on cancer cells by inhibiting proteasome activity. Subsequently, we hypothesized that 


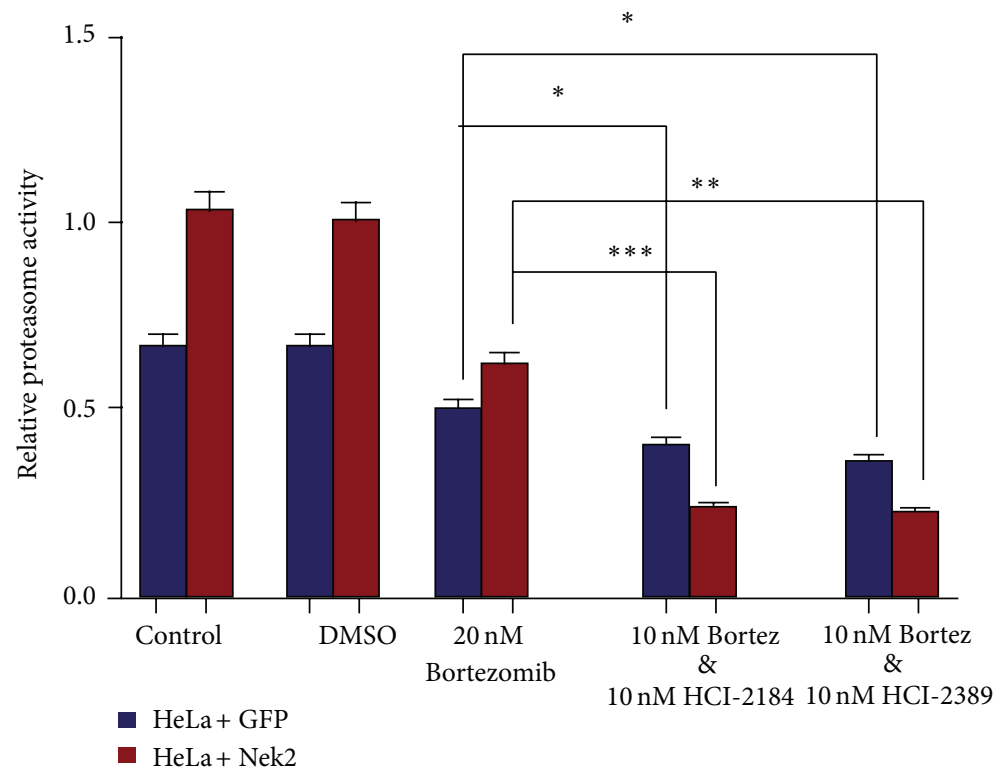

(a)
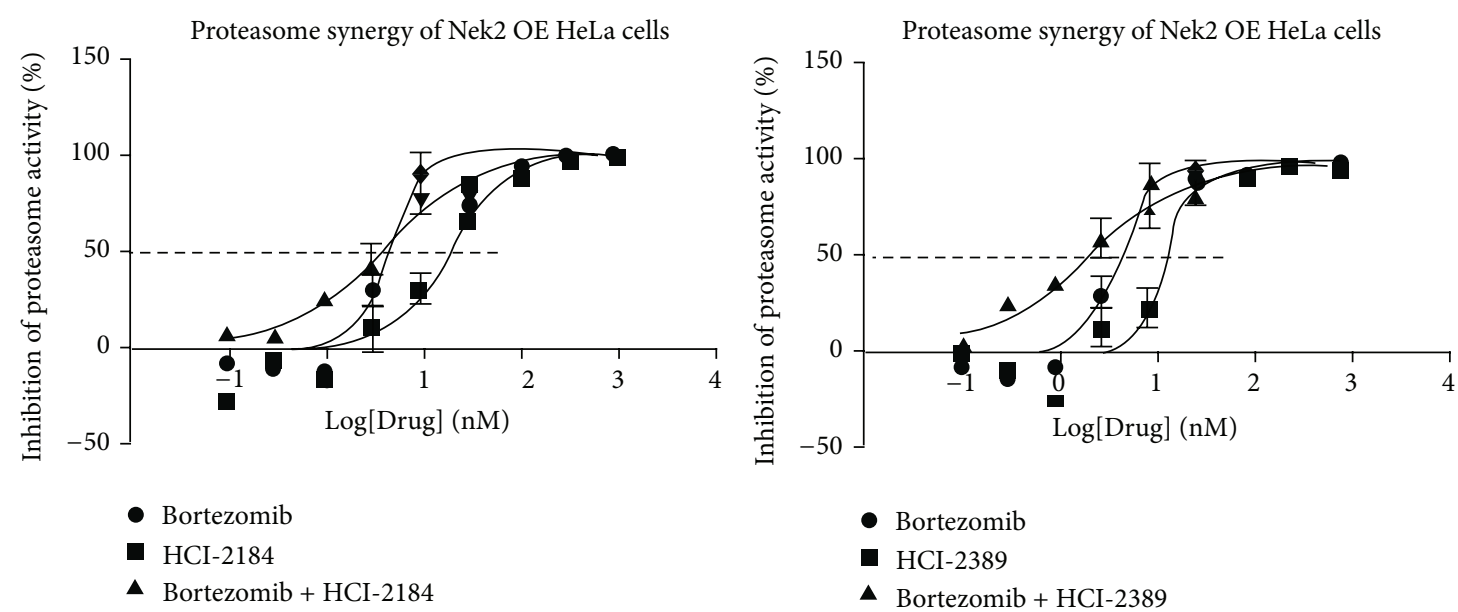

(b)

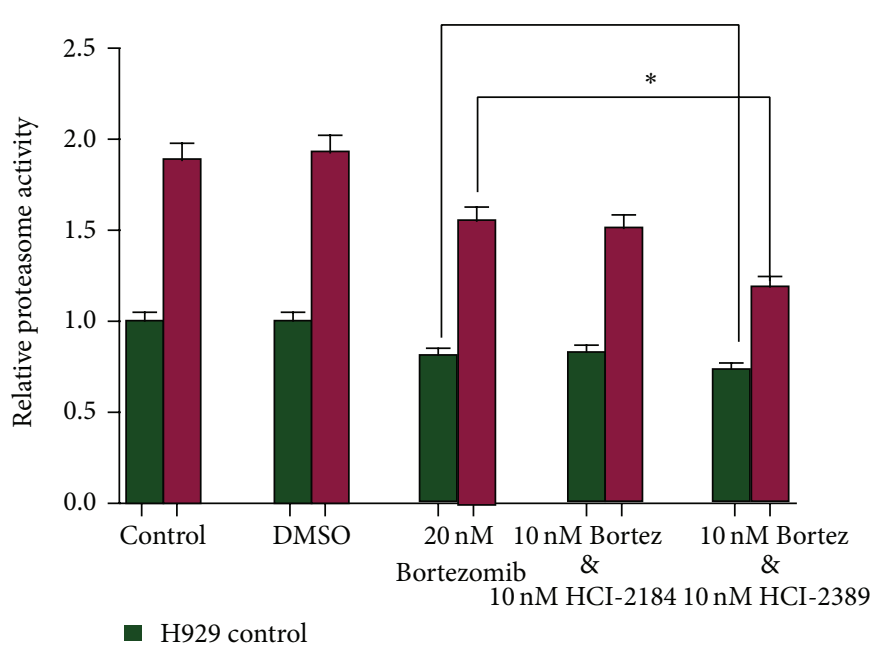

(c)

FIgure 6: Continued. 


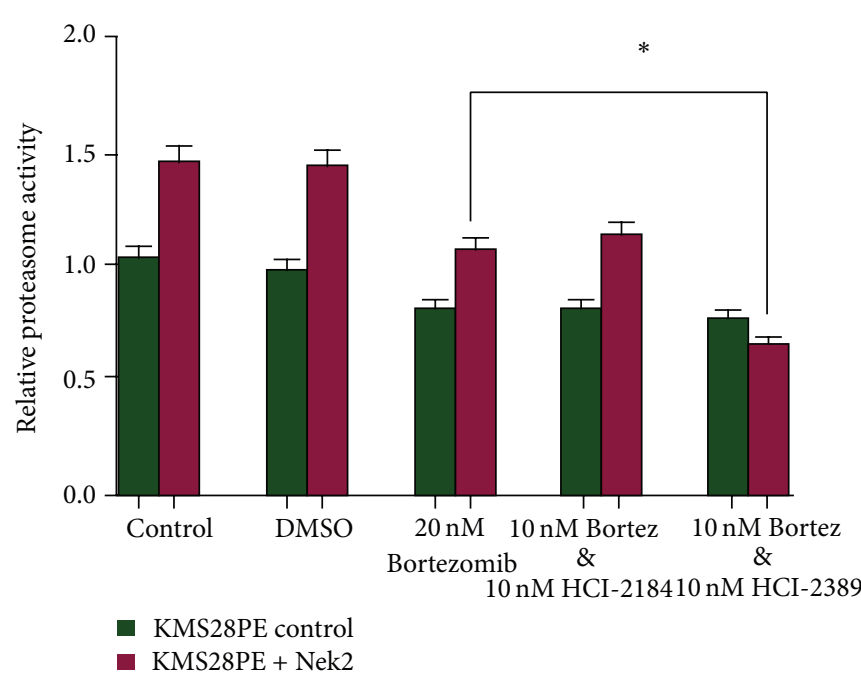

(d)

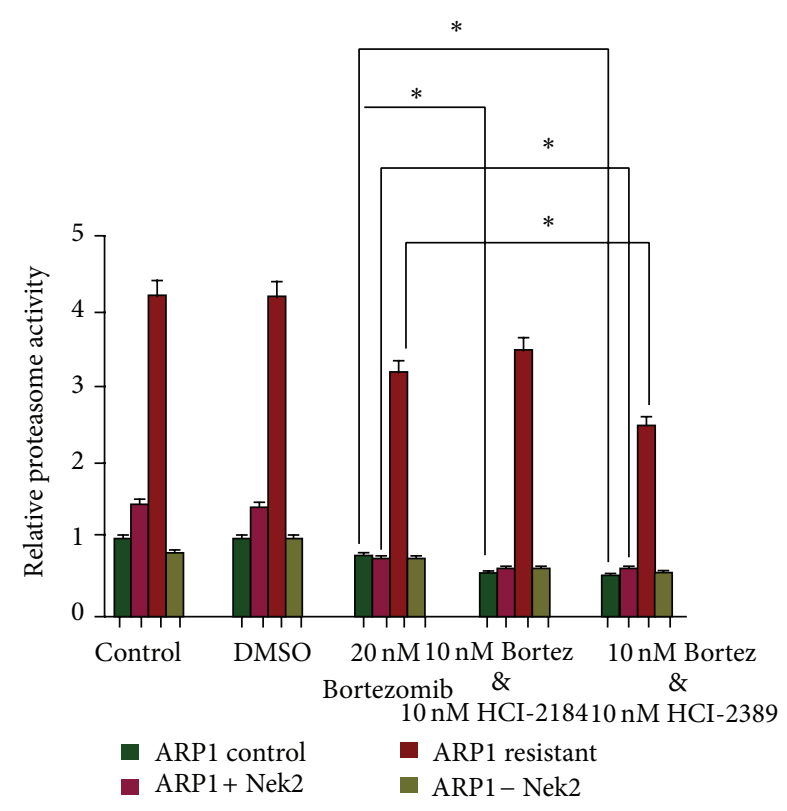

(e)

FIgURE 6: The combination of bortezomib and Nek2 inhibitors reduces proteasome activity to a greater extent than either drug alone. (a) The combination of bortezomib with either HCI-2184 or HCI-2389 significantly increased the effectiveness of bortezomib on Nek2-OE HeLa cells compared to GFP-transfected controls. (b) The combination of Nek2 inhibitors HCI-2184 or HCI-2389 and bortezomib inhibited proteasome activity in Nek2-OE HeLa cells to a greater extent than either drug alone. (c) and (d), the combination of Bortezomib and HCI-2389 decreased proteasome activity compared to untreated or DMSO treated H929 (c) or KMS28PE (d) cells treated with either empty vector (control) or Nek2 overexpressing (+Nek2) cells. (e), the combination of bortezomib and HCI-2389 decreased proteasome activity in empty vector (control), Nek2 plasmid (+Nek2), and Nek2 siRNA knockdown (-Nek2) ARP1 cells. The combination also resulted in a significant decrease in proteasome activity in ARP1 cells resistant to bortezomib (ARP1 resistant). For (a) and (c) $-(\mathrm{e}),{ }^{*} P<0.05,{ }^{* *} P<0.001,{ }^{* * *} P<0.0001$.

Nek2's role in bortezomib resistance was related to increasing proteasome activity. Using multiple cancer cell lines, we showed that overexpression of Nek2 significantly elevated proteasome activity. Specifically, we found higher proteasome activity in bortezomib resistant ARP1 cells. This elevated in vitro proteasome activity is inhibited by our Nek2 inhibitors HCI-2184 and HCI-2389 which rescue drug resistance of Nek2-OE HeLa cells. However, the mechanism of how Nek2 regulates proteasome activity is still unknown and needs further investigation.

Together with the Polo and Aurora kinase families, the NIMA-related protein kinases (Neks) have been called the third family of mitotic kinases [2]. Previous studies suggest that Nek family members influence cell cycle progression by regulating Cyclin B and Cdc2 [2, 49, 50]. Here, we discovered Nek2 overexpression down-regulated both Cyclin B and $\mathrm{Cdc} 2$ by increasing activity of the proteasome. This finding may provide more information for further study of Nek2's function in the cell cycle regulation.

By examining the proteasome activity of multiple cancer cell lines, we have identified Nek2 upregulation as a potential mechanism for bortezomib resistance related to proteasome activity elevation. However, for ARP1 cells, the proteasome activity of the bortezomib resistant clone was higher than that of the Nek2 OE clone (Figure 2(c)). Therefore, other mechanisms aside from Nek2 upregulation may be involved in proteasome activity elevation. Further effort is needed to elucidate other proteasome regulators as potential drug targets for MM therapeutics.

Although we have synthesized several potent Nek2 inhibitors with demonstrated activity (Figure 3), these inhibitors need better selectivity to advance them as potential clinical candidates (Supplementary Figure S2).

In summary, we have discovered that high levels of Nek2 expression are at least partly responsible for elevated proteasome activity and subsequent bortezomib resistance in human MM treatment. More excitingly, we have shown that Nek2 inhibition results in proteasome activity suppression and cell cycle arrest. This research provides important knowledge for future studies of Nek2's biological function and provides potential solutions for bortezomib resistance in MM therapy.

\section{Clinical Practice Points}

Although progress in the treatment of MM has been made in the past decade $[40,41]$, myeloma remains largely incurable with current therapeutic strategies. Bortezomib is one of the most effective chemotherapies for MM, but drug resistance remains a crucial problem with bortezomib treatment. Little is known about the molecular mechanisms involved in this 

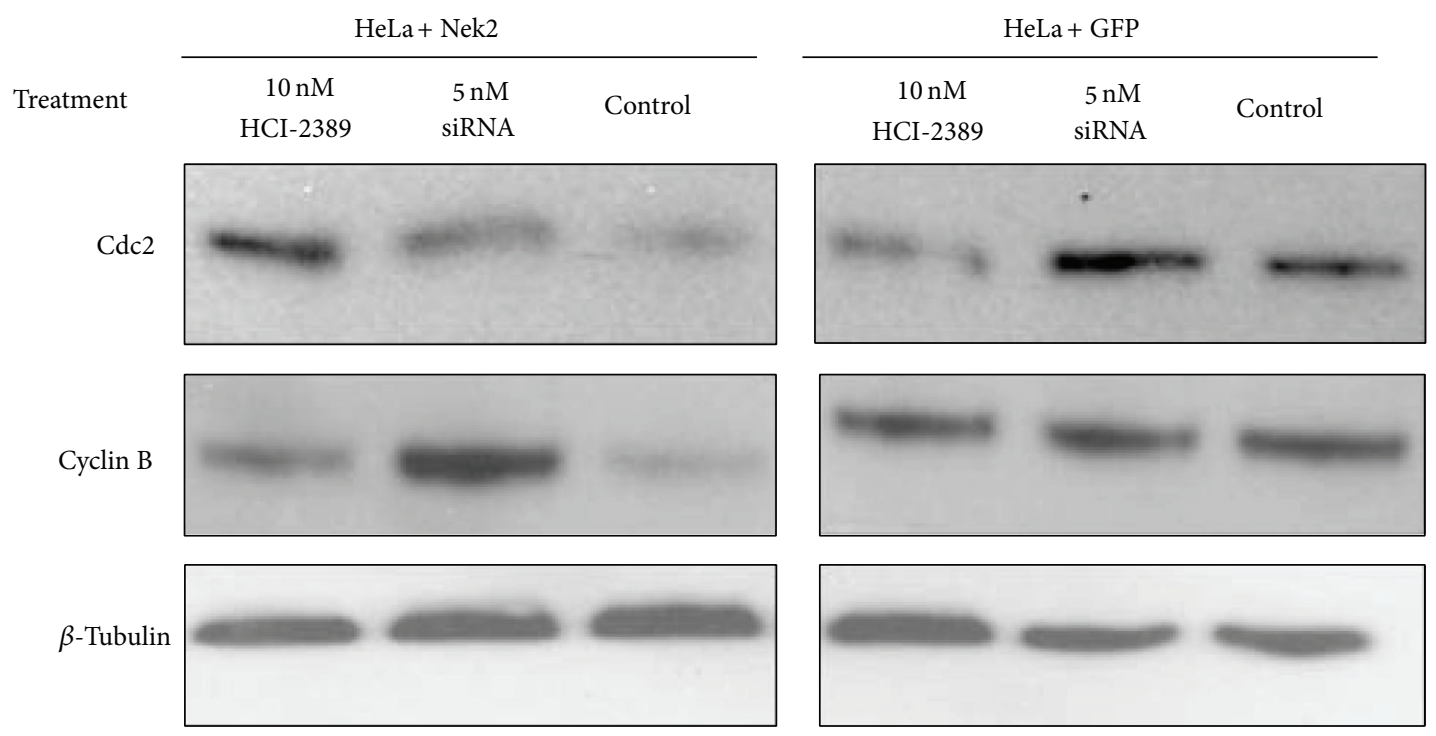

(a)
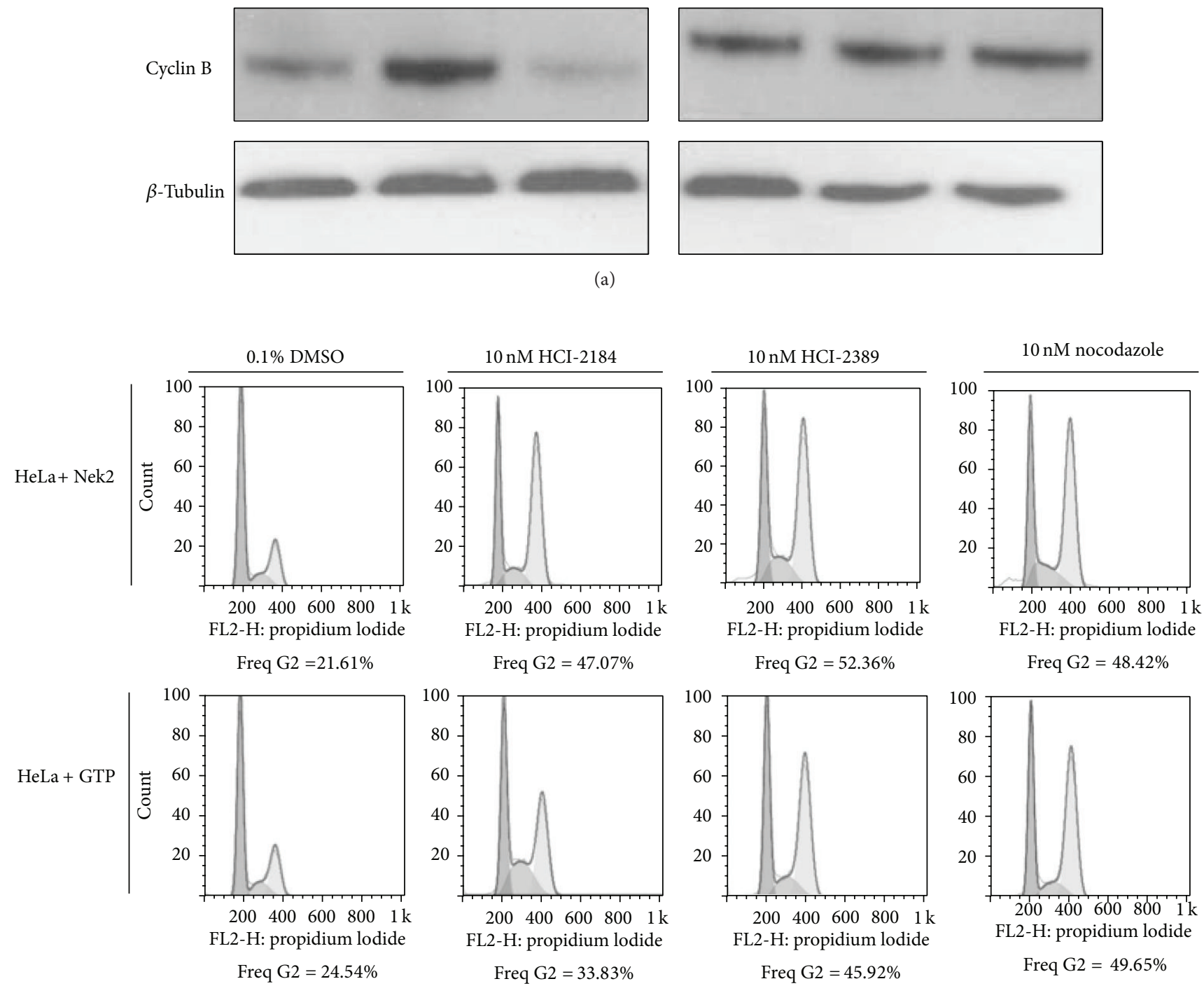

FL2-H: propidium lodide

Freq G2 $=52.36 \%$

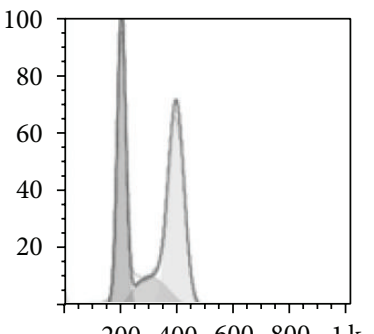

$200400600 \quad 800 \quad 1 \mathrm{k}$ FL2-H: propidium lodide

Freq G2 $=45.92 \%$

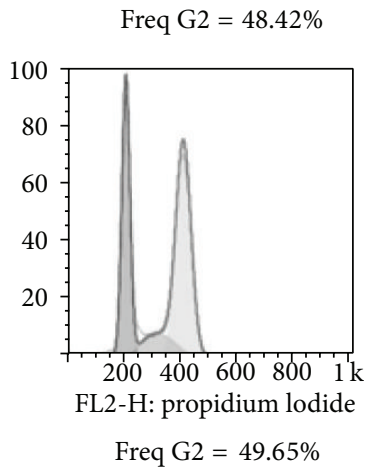

(b)

FIGURE 7: Novel Nek2 inhibitor prevents Cdc2 and Cyclin B from being degraded by the proteasome, catching HeLa cells in G2/M phase. (a) Western blot showed that the degradation of Cdc2 and Cyclin B was inhibited by treatment of $10 \mathrm{nM} \mathrm{HCI}-2389$. This effect was significant in Nek2 overexpressed HeLa cells. (b) Flow cytometry data showed that, treated by $10 \mathrm{nM} \mathrm{HCI}-2184$ or $10 \mathrm{nM} \mathrm{HCI}-2389$, around $50 \%$ HeLa cells stayed at G2/M phase. $100 \mathrm{nM}$ nocodazole was used as the positive control.

resistance. In our previous report, we used gene expression profiling in a variety of MM cases and identified Nek2 as the most significant gene associated with early relapse. Other reports have similarly shown that Nek 2 overexpression induces chemotherapeutic resistance in vitro. It is clear that there is an urgent need for exploring the mechanism linking the Nek2 kinase to drug resistance and the development of novel Nek2 inhibitors. To our knowledge, this study represents the first link connecting Nek2's biological function of regulating proteasome activity as the mechanism of bortezomib resistance in multiple myeloma. It is also the first to establish highly effective Nek2 inhibitors 
that successfully inhibit proteasome activity in cancer cell lines.

\section{Conflict of Interests}

Lingyao Meng and Alexis Mollard were previously employed by Tolero Pharmaceuticals Inc. Kent Carpenter is currently employed by Tolero Pharmaceuticals Inc. Steven Warner is currently employed by Tolero Pharmaceuticals Inc. David Bearss is the CEO of Tolero Pharmaceuticals Inc. All other authors state that they have no potential conflict of interests.

\section{Acknowledgments}

This research is funded by the NIH Grant NIH-R01-CA152105 and the Leukemia Lymphoma Society TRP (624611). Additional funds were attained privately through Tolero Pharmaceuticals Inc.

\section{References}

[1] A. M. Fry, "The Nek2 protein kinase: a novel regulator of centrosome structure," Oncogene, vol. 21, no. 40, pp. 6184-6194, 2002.

[2] L. Wu, S. A. Osmani, and P. M. Mirabito, "A role for NIMA in the nuclear localization of cyclin B in Aspergillus nidulans," Journal of Cell Biology, vol. 141, no. 7, pp. 1575-1587, 1998.

[3] H. Rajagopalan and C. Lengauer, "Aneuploidy and cancer," Nature, vol. 432, pp. 338-341, 2004.

[4] T. Fujioka, Y. Takebayashi, M. Ito, and T. Uchida, "Nek2 expression and localization in porcine oocyte during maturation," Biochemical and Biophysical Research Communications, vol. 279, no. 3, pp. 799-802, 2000.

[5] A. Grallert and I. M. Hagan, "Schizosaccharomyces pombe NIMA-related kinase, Finl, regulates spindle formation and an affinity of Polo for the SPB," The EMBO Journal, vol. 21, no. 12, pp. 3096-3107, 2002.

[6] D. Patel, A. Incassati, N. Wang, and D. J. McCance, "Human papillomavirus type 16 E6 and E7 cause polyploidy in human keratinocytes and up-regulation of $\mathrm{G}_{2}-\mathrm{M}$-phase proteins," Cancer Research, vol. 64, no. 4, pp. 1299-1306, 2004.

[7] Y. H. Kim, J. Y. Choi, Y. Jeong, D. J. Wolgemuth, and K. Rhee, "Nek2 localizes to multiple sites in mitotic cells, suggesting its involvement in multiple cellular functions during the cell cycle," Biochemical and Biophysical Research Communications, vol. 290, no. 2, pp. 730-736, 2002.

[8] T. Mayor, U. Hacker, Y.-D. Stierhof, and E. A. Nigg, "The mechanism regulating the dissociation of the centrosomal protein C-Napl from mitotic spindle poles," Journal of Cell Science, vol. 115, no. 16, pp. 3275-3284, 2002.

[9] N. R. Helps, X. Luo, H. M. Barker, and P. T. W. Cohen, "NIMArelated kinase 2 (Nek2), a cell-cycle-regulated protein kinase localized to centrosomes, is complexed to protein phosphatase 1," Biochemical Journal, vol. 349, part 2, pp. 509-518, 2000.

[10] A. M. Fry, P. Meraldi, and E. A. Nigg, "A centrosomal function for the human Nek2 protein kinase, a member of the NIMA family of cell cycle regulators," The EMBO Journal, vol. 17, no. 2, pp. 470-481, 1998.

[11] J. Codony-Servat, M. A. Tapia, M. Bosch et al., "Differential cellular and molecular effects of bortezomib, a proteasome inhibitor, in human breast cancer cells," Molecular Cancer Therapeutics, vol. 5, no. 3, pp. 665-675, 2006.

[12] M. D. Jones, J. C. Liu, T. K. Barthel et al., "A proteasome inhibitor, bortezomib, inhibits breast cancer growth and reduces osteolysis by downregulating metastatic genes," Clinical Cancer Research, vol. 16, no. 20, pp. 4978-4989, 2010.

[13] R. Z. Orlowski and E. C. Dees, "The role of the ubiquitinationproteasome pathway in breast cancer: applying drugs that affect the ubiquitin-proteasome pathway to the therapy of breast cancer," Breast Cancer Research, vol. 5, no. 1, pp. 1-7, 2003.

[14] X. Zeng, F. Y. Shaikh, M. K. Harrison et al., “The Ras oncogene signals centrosome amplification in mammary epithelial cells through cyclin D1/Cdk4 and Nek2," Oncogene, vol. 29, no. 36, pp. 5103-5112, 2010.

[15] N. Tsunoda, T. Kokuryo, K. Oda et al., "Nek2 as a novel molecular target for the treatment of breast carcinoma," Cancer Science, vol. 100, no. 1, pp. 111-116, 2009.

[16] G. Wu, X. L. Qiu, L. Zhou et al., "Small molecule targeting the Hec1/Nek2 mitotic pathway suppresses tumor cell growth in culture and in animal," Cancer Research, vol. 68, no. 20, pp. 8393-8399, 2008.

[17] K. Ahmad, "Proteasome inhibitor for treatment of multiple myeloma," The Lancet Oncology, vol. 6, no. 8, p. 546, 2005.

[18] D. Chauhan, T. Hideshima, and K. C. Anderson, "Proteasome inhibition in multiple myeloma: therapeutic implication," Annual Review of Pharmacology and Toxicology, vol. 45, pp. 465-476, 2005.

[19] U. Testa, "Proteasome inhibitors in cancer therapy," Current Drug Targets, vol. 10, no. 10, pp. 968-981, 2009.

[20] I. Zavrski, L. Kleeberg, M. Kaiser et al., "Proteasome as an emerging therapeutic target in cancer," Current Pharmaceutical Design, vol. 13, no. 5, pp. 471-485, 2007.

[21] W. Zhou, Y. Yang, J. Xia et al., "NEK2 induces drug resistance mainly through activation of efflux drug pumps and is associated with poor prognosis in myeloma and other cancers," Cancer Cell, vol. 23, no. 1, pp. 48-62, 2013.

[22] M. Eto, E. Elliott, T. D. Prickett, and D. L. Brautigan, "Inhibitor-2 regulates protein phosphatase-1 complexed with NimA-related kinase to induce centrosome separation," Journal of Biological Chemistry, vol. 277, no. 46, pp. 44013-44020, 2002.

[23] P. Rellos, F. J. Ivins, J. E. Baxter et al., "Structure and regulation of the human Nek2 centrosomal kinase," The Journal of Biological Chemistry, vol. 282, no. 9, pp. 6833-6842, 2007.

[24] M. Li, D. L. Satinover, and D. L. Brautigan, "Phosphorylation and functions of inhibitor-2 family of proteins," Biochemistry, vol. 46, no. 9, pp. 2380-2389, 2007.

[25] I. Westwood, D. M. Cheary, J. E. Baxter et al., "Insights into the conformational variability and regulation of human Nek2 kinase," Journal of Molecular Biology, vol. 386, no. 2, pp. 476485, 2009.

[26] G. Nikcevic, N. Kovacevic-Grujicic, and M. Stevanovic, "Improved transfection efficiency of cultured human cells," Cell Biology International, vol. 27, no. 9, pp. 735-737, 2003.

[27] M. N. Malik, W. D. Spivack, A. M. Sheikh, and M. D. Fenko, "The 26S proteasome in garlic (Allium sativum): purification and partial characterization," Journal of Agricultural and Food Chemistry, vol. 52, no. 11, pp. 3350-3355, 2004.

[28] S. W. Sherwood and R. T. Schimke, "Duality in DC programming: the case of several constraints," Methods in Cell Biology, vol. 46, pp. 77-97, 1995. 
[29] J. Mi, C. Guo, D. L. Brautigan, and J. M. Larner, "Protein phosphatase- $1 \alpha$ regulates centrosome splitting through Nek2," Cancer Research, vol. 67, no. 3, pp. 1082-1089, 2007.

[30] P. G. Richardson, T. Hideshima, and K. C. Anderson, "Bortezomib (PS-341): a novel, first-in-class proteasome inhibitor for the treatment of multiple myeloma and other cancers," Cancer Control, vol. 10, no. 5, pp. 361-369, 2003.

[31] N. Bader, T. Jung, and T. Grune, "The proteasome and its role in nuclear protein maintenance," Experimental Gerontology, vol. 42, no. 9, pp. 864-870, 2007.

[32] A. Dinarina, P. G. Santamaria, and A. R. Nebreda, "Cell cycle regulation of the mammalian CDK activator RINGO/Speedy A," FEBS Letters, vol. 583, no. 17, pp. 2772-2778, 2009.

[33] A. D. DeWard and A. S. Alberts, "Ubiquitin-mediated degradation of the formin mDia2 upon completion of cell division," Journal of Biological Chemistry, vol. 284, no. 30, pp. 2006120069, 1945.

[34] S. I. Reed, "The ubiquitin-proteasome pathway in cell cycle control," Results and Problems in Cell Differentiation, vol. 42, pp. 147-181, 2006.

[35] S. S. Margolis, J. A. Perry, D. H. Weitzel et al., "A role for PP1 in the Cdc2/Cyclin B-mediated positive feedback activation of Cdc25," Molecular Biology of the Cell, vol. 17, no. 4, pp. 1779-1789, 2006.

[36] J.-Y. Ji, J. Crest, and G. Schubiger, "Genetic interactions between Cdk1-CyclinB and the separase complex in Drosophila," Development, vol. 132, no. 8, pp. 1875-1884, 2005.

[37] B. B. Olsen and B. Guerra, "Ability of CK2beta to selectively regulate cellular protein kinases," Molecular and Cellular Biochemistry, vol. 316, no. 1-2, pp. 115-126, 2008.

[38] P. Chadebech, I. Truchet, L. Brichese, and A. Valette, "Upregulation of cdc2 protein during paclitaxel-induced apoptosis," International Journal of Cancer, vol. 87, no. 6, pp. 779-786, 2000.

[39] Y. Chen, Z. D. Sharp, and W.-H. Lee, "HEC binds to the seventh regulatory subunit of the $26 \mathrm{~S}$ proteasome and modulates the proteolysis of mitotic cyclins," The Journal of Biological Chemistry, vol. 272, no. 38, pp. 24081-24087, 1997.

[40] B. Barlogie, G. Tricot, E. Anaissie et al., "Thalidomide and hematopoietic-cell transplantation for multiple myeloma," New England Journal of Medicine, vol. 354, no. 10, pp. 1021-1030, 2006.

[41] J. A. Child, G. J. Morgan, F. E. Davies et al., "High-dose chemotherapy with hematopoietic stem-cell rescue for multiple myeloma," New England Journal of Medicine, vol. 348, no. 19, pp. 1875-1883, 2003.

[42] X. Liu, Y. Gao, Y. Lu, J. Zhang, L. Li, and F. Yin, "Upregulation of NEK2 is associated with drug resistance in ovarian cancer," Oncology Reports, vol. 31, no. 2, pp. 745-754, 2014.

[43] H. S. Ko, T. Uehara, K. Tsuruma, and Y. Nomura, "Ubiquilin interacts with ubiquitylated proteins and proteasome through its ubiquitin-associated and ubiquitin-like domains," FEBS Letters, vol. 566, no. 1-3, pp. 110-114, 2004.

[44] R. J. Mayer and J. Fujita, "Gankyrin, the 26 S proteasome, the cell cycle and cancer," Biochemical Society Transactions, vol. 34, no. 5, pp. 746-748, 2006.

[45] D. J. McConkey and K. Zhu, "Mechanisms of proteasome inhibitor action and resistance in cancer," Drug Resistance Updates: Reviews and Commentaries in Antimicrobial and Anticancer Chemotherapy, vol. 11, no. 4-5, pp. 164-179, 2008.

[46] N. Watanabe, H. Arai, J.-I. Iwasaki et al., "Cyclin-dependent kinase (CDK) phosphorylation destabilizes somatic Weel via multiple pathways," Proceedings of the National Academy of Sciences of the United States of America, vol. 102, no. 33, pp. 11663-11668, 2005.

[47] J. R. Skaar and M. Pagano, "Control of cell growth by the SCF and APC/C ubiquitin ligases," Current Opinion in Cell Biology, vol. 21, no. 6, pp. 816-824, 2009.

[48] S. Tudzarova, S. L. Colombo, K. Stoeber, S. Carcamo, G. H. Williams, and S. Moncada, "Two ubiquitin ligases, APC/CCdh1 and SKP1-CUL1-F (SCF)- $\beta$-TrCP, sequentially regulate glycolysis during the cell cycle," Proceedings of the National Academy of Sciences of the United States of America, vol. 108, no. 13, pp. 5278-5283, 2011.

[49] J. He, J. Xu, X. X. Xu, and R. A. Hall, "Cell cycle-dependent phosphorylation of disabled-2 by cdc2," Oncogene, vol. 22, no. 29, pp. 4524-4530, 2003.

[50] K. J. Stanya, Y. Liu, A. R. Means, and H. Y. Kao, "Cdk2 and Pinl negatively regulate the transcriptional corepressor SMRT," Journal of Cell Biology, vol. 183, no. 1, pp. 49-61, 2008. 

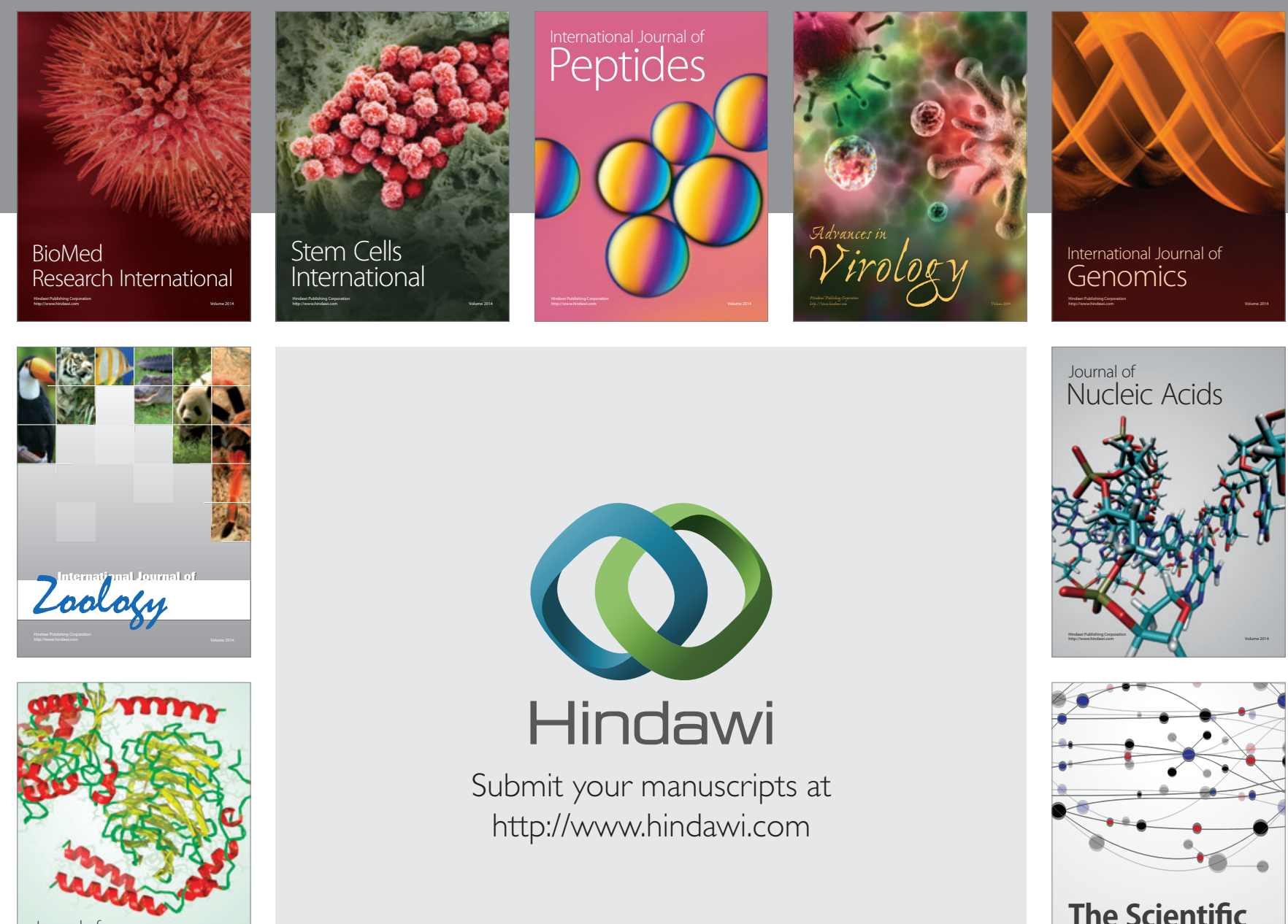

Submit your manuscripts at

http://www.hindawi.com

Journal of
Signal Transduction
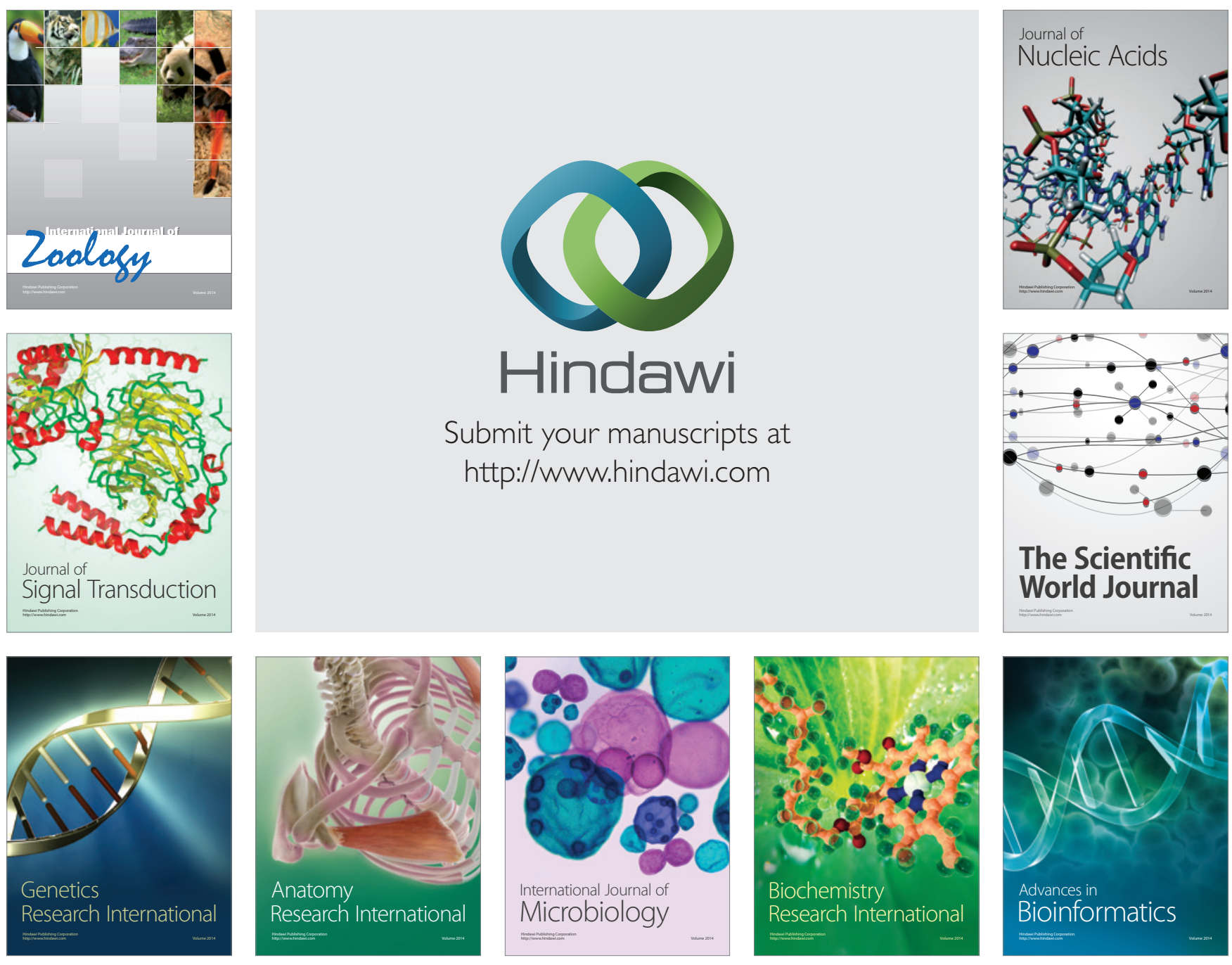

The Scientific World Journal
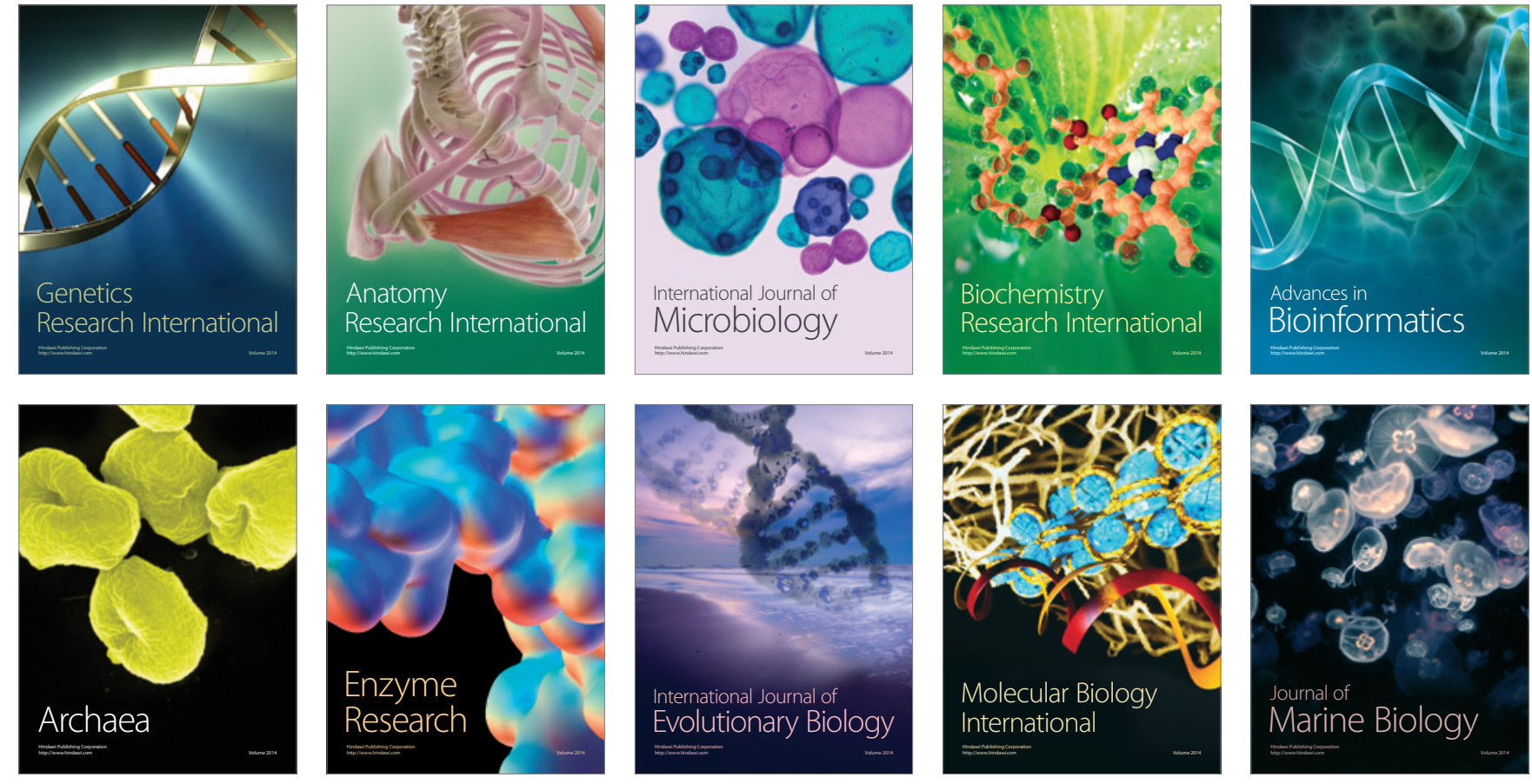